\title{
Calorimeter Simulation with Hadrons in CMS
}

\author{
Stefan Piperov \\ Institute for Nuclear Research and Nuclear Energy. \\ Bulgarian Academy of Sciences. Sofia, Bulgaria \\ and \\ Fermilab, Batavia, Illinois, USA
}

\begin{abstract}
CMS is using Geant4 to simulate the detector setup for the forthcoming data from the LHC. Validation of physics processes inside Geant4 is a major concern in view of getting a proper description of jets and missing energy for signal and background events. This is done by carrying out an extensive studies with test beam using the prototypes or real detector modules of the CMS calorimeter. These data are matched with Geant4 predictions using the same framework that is used for the entire CMS detector. Tuning of the Geant4 models is carried out and steps to be used in reproducing detector signals are defined in view of measurements of energy response, energy resolution, transverse and longitudinal shower profiles for a variety of hadron beams over a broad energy spectrum between 2 to $300 \mathrm{GeV} / \mathrm{c}$. The tuned Monte Carlo predictions match many of these measurements within systematic uncertainties.
\end{abstract}

\section{INTRODUCTION}

When hadrons go through matter they lose their energy and identity in interactions with the medium, creating showers of new particles who follow the same fate until finally all the energy of the initial hadron gets deposited in the medium. By measuring the energy deposited in the medium one can estimate the energy of the initial hadron. This is how calorimeters work.

The fraction of particle's energy lost through electromagnetic processes creates an electromagnetic shower, while the fraction lost through hadronic processes creates a hadronic shower. Electromagnetic showers, due to the relative simplicity of the involved interactions, are very well understood and modeled in Monte-Carlo simulations with great accuracy. Hadronic showers, on the other hand, are not so well modeled in simulations yet, and are subject of active investigation in recent years. Because of the uncertainties in the hadronic shower simulations, and also to save computing time - since tracking of thousands of particles in the calorimeter material can be quite CPU-intensive job - sometimes parametrized hadronic showers are used in place of detail simulated ones. These parametrization are not necessarily based on the detailed simulations for the above mentioned reasons.

The CMS collabotarion has taken the challenge to provide detailed hadronic shower simulation, in agreement with the measured parameters of the calorimetric system, and to base the faster, parametrized shower simulation on this detailed simulation.

With the latest advances in simulation codes like e.g. the Geant4 Toolkit, the Monte-Carlo predictions of calorimeters' performance for hadronic showers get in much better

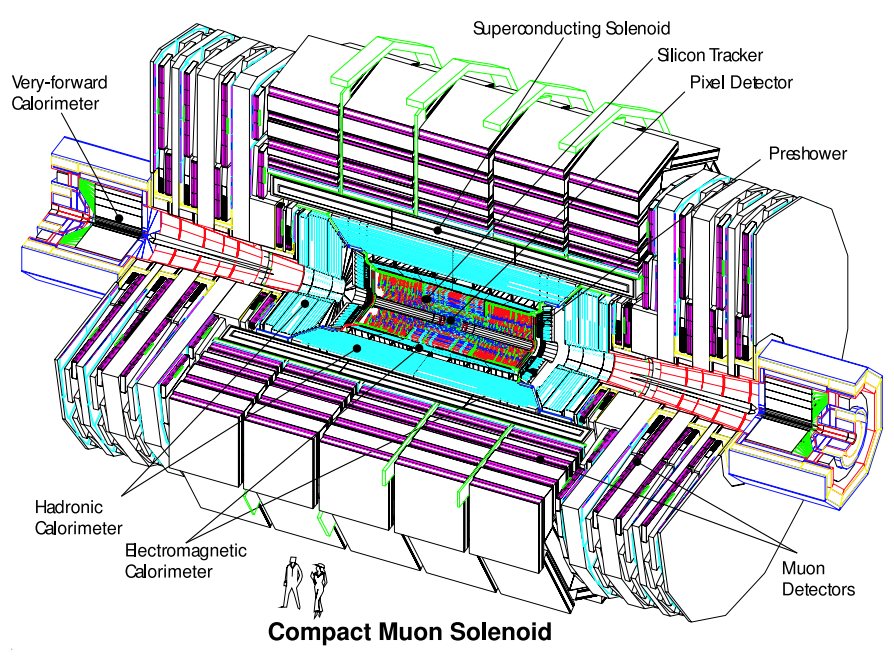

Fig. 1. The CMS detector

agreement with the performance measured in test-beams. The current work shows the degree of this agreement for the CMS [1], [2] detector's calorimetric system, as measured in the 2004 and 2006 test-beams conducted on SPS's H2 beamline at CERN, and simulated with several versions of Geant4.

\section{CMS DETECTOR AND ITS CALORIMETRIC SYSTEM}

Fig. 1 shows the CMS detector with its major sub-detectors. With the exception of the Very-Forward and Hadron Outer

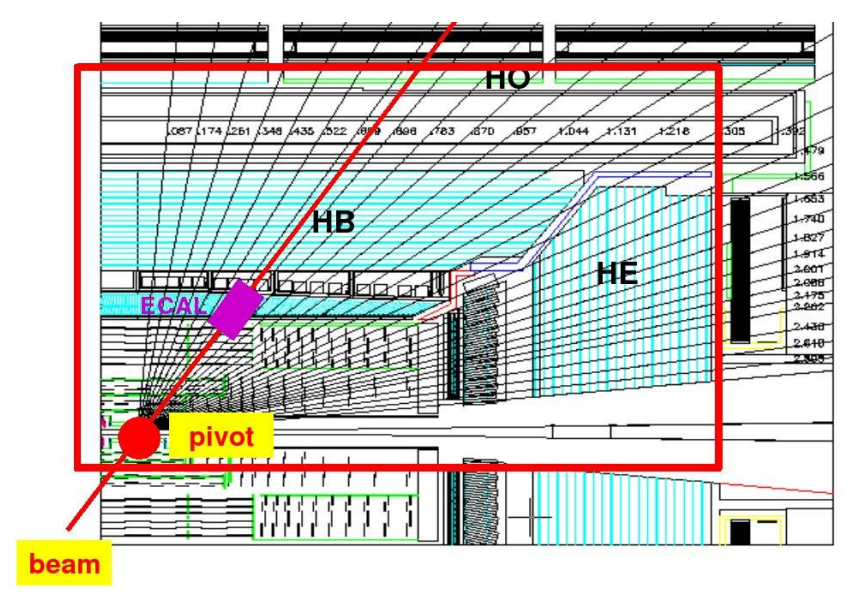

Fig. 2. H2 setup: detectors present on the testbeam table. Pivot point corresponds to beam interaction point in CMS 


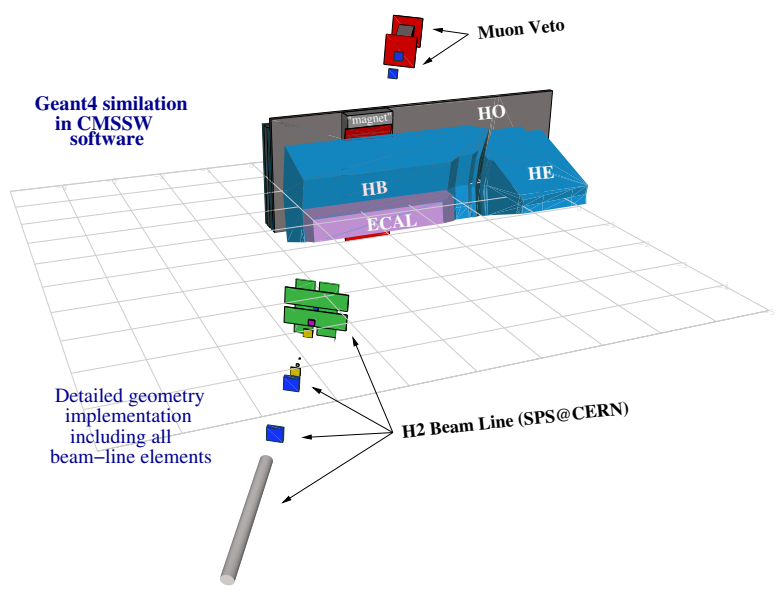

Fig. 3. Detailed simulation geometry of the setup, visualized in IGUANA

calorimeters, the calorimetric system of CMS is contained within the superconducting solenoidal magnet. Fig.2 shows the section of the calorimetric system present on the testbeam table in the $\mathrm{H} 2$ beamline. Two wedges of the Hadronic Calorimeter (HCAL) Barrel part (HB), one wedge of HCAL Endcap (HE), Two layers of HCAL Outer (HO), and one super-module of Electromagnetic calorimeter (ECAL) crystals are mounted on a table allowing for rotation in both eta- and phi-direction with respect to the $\mathrm{H} 2$ beamline, thus allowing for single-particle beam to be sent to any part of the calorimetric system. The pivot point of the table corresponds to the beam interaction point in CMS.

The setup is complemented with a set of beam-line elements, allowing for particle identification - Time-Of-Flight system, Cherenkov detectors, Muon-veto system; beam cleaning and triggering - Beam Scintillator Counters, Beam Halo Counters; and precise beam position measurement - a set of proportional wire-chambers. All this provides a very clean, single-particle hadron beam with beam momenta from 2 to $350 \mathrm{GeV} / \mathrm{c}$.

\section{Monte-Carlo Simulation}

The Monte-Carlo simulation of the experimental setup was done using various versions of the Geant4 Toolkit within the CMS software framework - CMSSW [5]. Every effort has been made to ensure that the simulation geometry follows exactly the geometry of the real detector, including all geometrical volumes and materials. A visualization of the simulation geometry, using IGUANA [6], is shown in Fig.3.

Various physics models of hadron interactions, as described in the physics reference manual [7], were used in the simulation. In the early simulations those included the LHEP and QGSP models, while in the later versions also the Bertinicascade models QGSP-BERT and QGSP-BERT-EMV were included.

\section{EARLY SIMULATIONS AND DISCREPANCIES WITH THE TEST-BEAM RESULTS}

The main areas of (macroscopic) comparison between the simulated and test-beam results were:

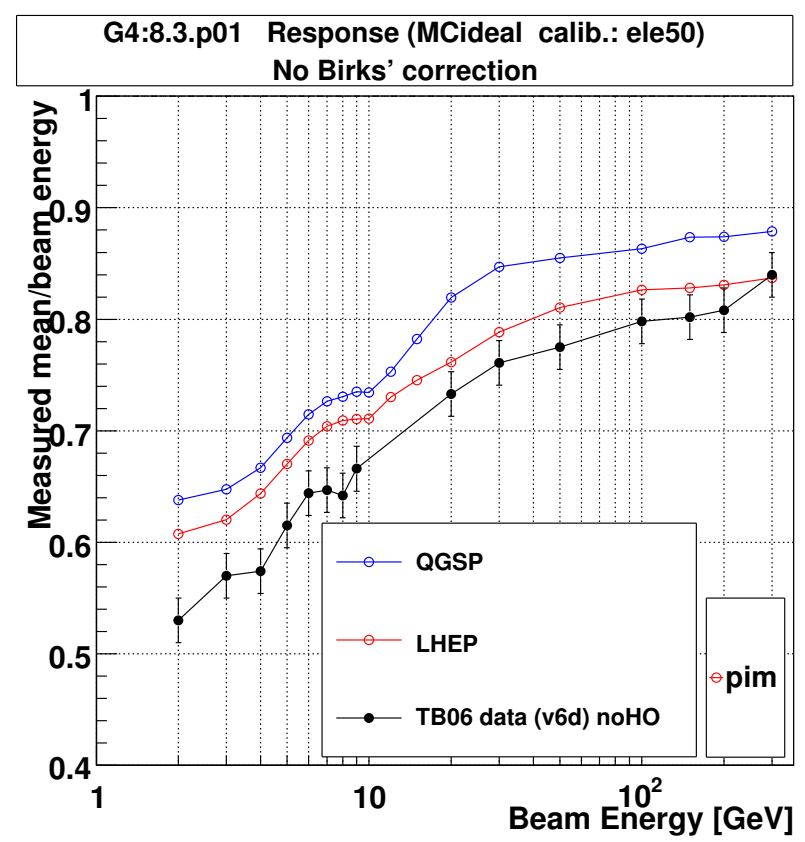

Fig. 6. Early Simulation: Linearity of response. Both physics models overestimate the response of the calorimeters at all beam momenta.

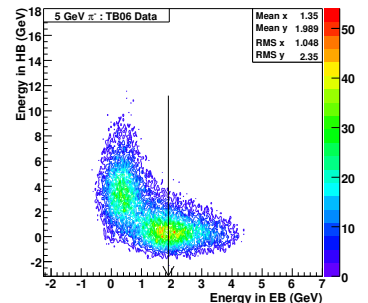

Test-beam

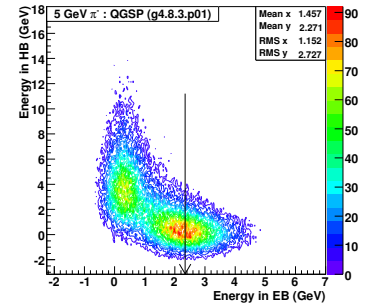

Simulation
Fig. 7. Early discrepancies: "banana" plot for $5 \mathrm{GeV}$ pion beam

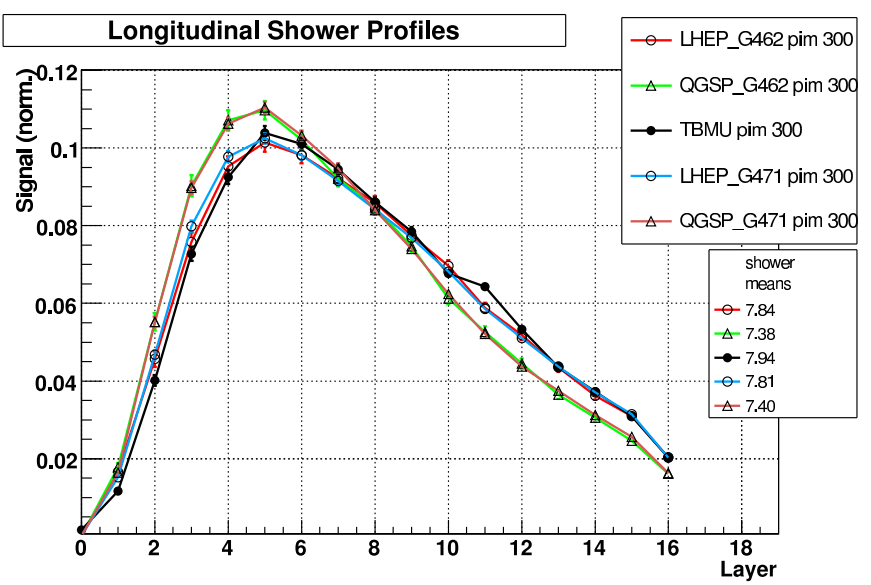

Fig. 8. Early Simulation: Longitudinal shower profile 


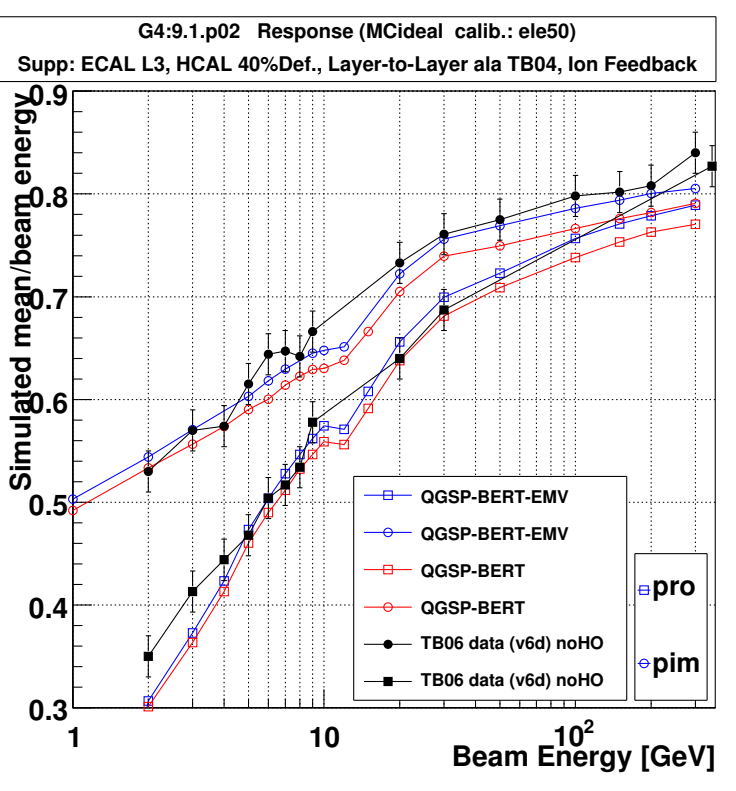

Combined ECAL+HCAL

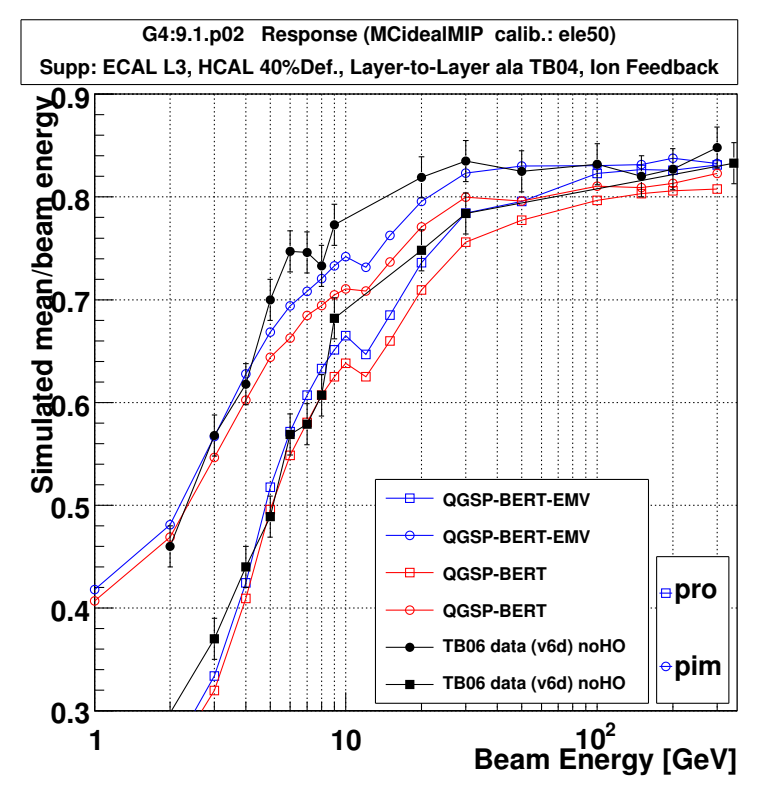

HCAL alone

Fig. 4. Improved Simulation: Linearity of response.

\section{G4:9.2.b01 Resolution (MCideal)}

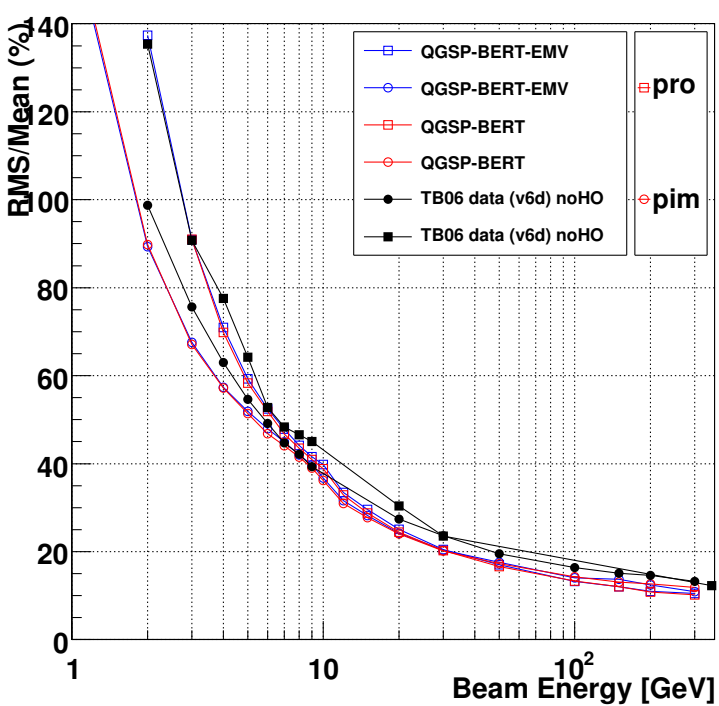

Combined ECAL+HCAL

\section{G4:9.2.b01 Resolution (MCidealMIP)}

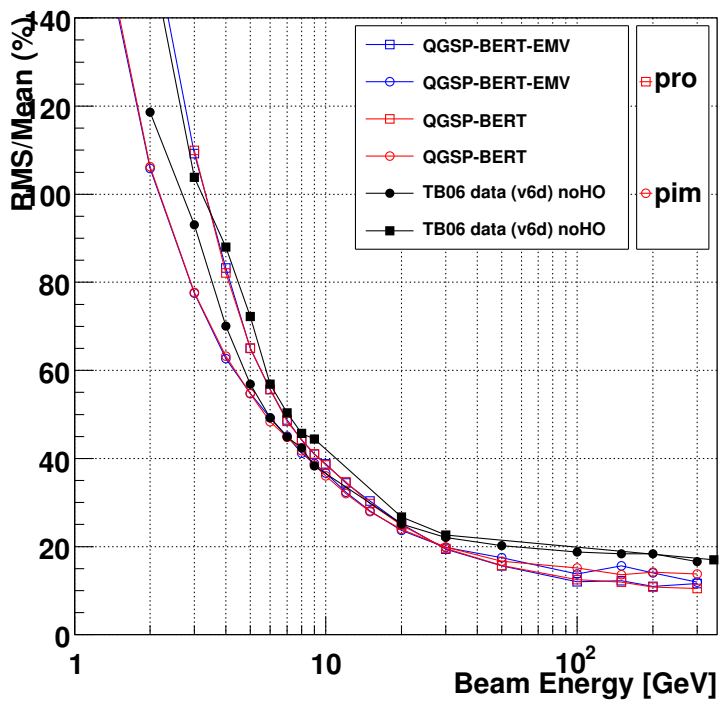

HCAL alone

Fig. 5. Improved Simulation: Resolution.

- linearity of response

- longitudinal and transverse shower profiles

In both simulation and test-beam case the calorimeters were independently calibrated to $50 \mathrm{GeV} / \mathrm{c}$ electron beams: a 50 $\mathrm{GeV} / \mathrm{c}$ electron beam sent into either ECAL or HCAL would result in $50 \mathrm{GeV} / \mathrm{c}$ response for the given detector. In this way any observed difference between the simulated and measured response would be due only to differences in the shower development.

Fig.6 shows the comparison of linearity of response in early (up to Geant 4 v.8.3) versions of the simulations to test- beam data. Both physics models LHEP and QGSP predicted systematically higher response of the calorimeters in pion beams. The same is shown in detail in Fig.7 in the so-called "banana" plot: reconstructed energy in HCAL vs. ECAL.

In addition, the longitudinal shower profile for highmomenta pions predicted by the QGSP model differed significantly from the measured one, as seen in Fig.8.

\section{IMPROVEMENTS TO THE SIMULATION}

Following these findings, the following improvements were done to the Monte-Carlo simulation: 


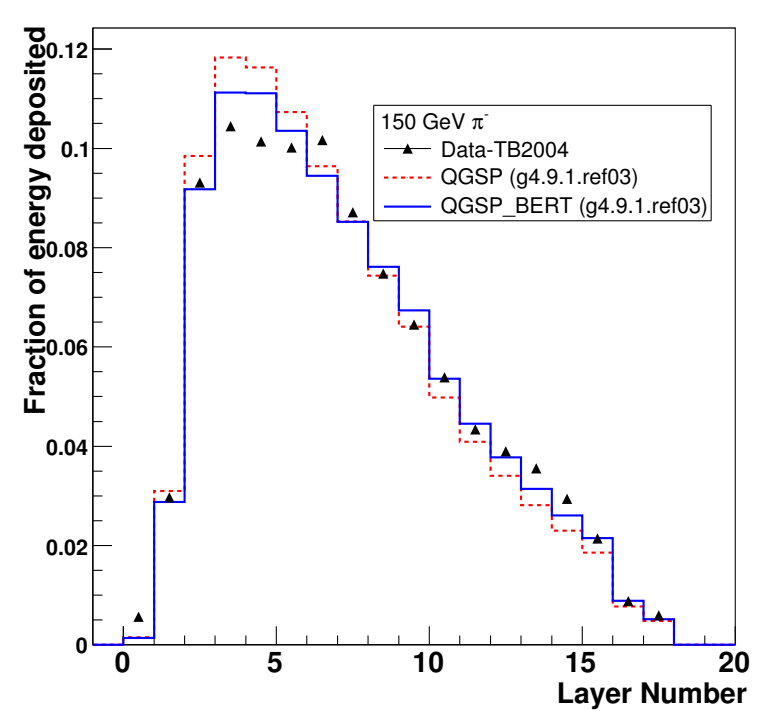

Longitudinal)

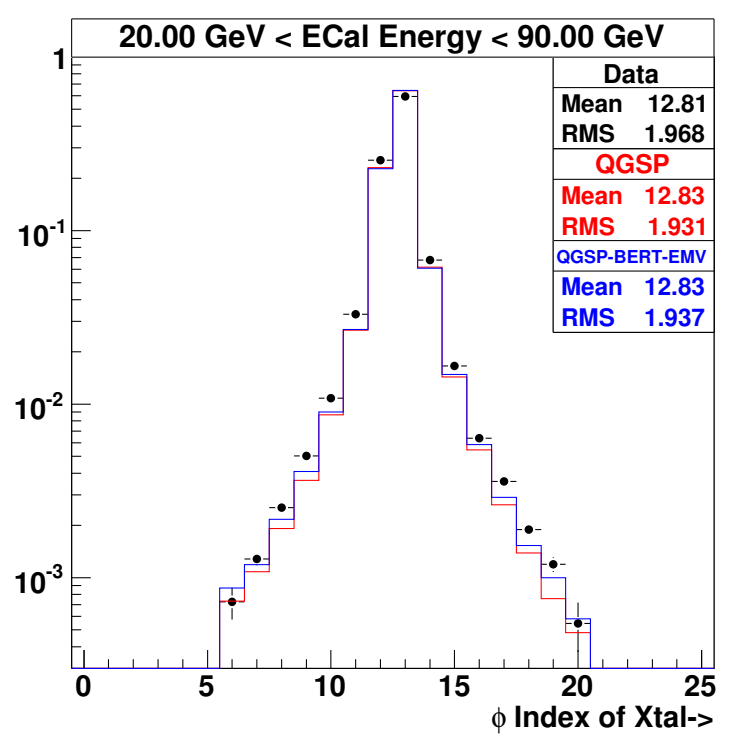

Transverse)

Fig. 9. Improved Simulation: Hadronic shower profiles.

- Introduce scintillation saturation effect through the use of Birks' law. Corresponding Birks' constants were extracted from optimization process.

- Use new physics models - QGSP-BERT and QGSPBERT-EMV. Both use Bertini intra-nuclear cascade for beam momenta below $10 \mathrm{GeV} / \mathrm{c}$, and just differ slightly in the treatment of multiple scattering.

- Feed the results back to the Geant4 collaboration, resulting in a new and improved version of the toolkit G4.9.1.p01 - with:

- Improved Bertini cascade code, including now Coulomb barrier in the de-excitation process.

- Better treatment of quasi-elastic scattering for high energy region of QGS model.

- changes in LHEP model for improved $p_{T}$ distribution of hadrons from strange baryon reactions.

- inclusion of electromagnetic interactions for longlived charged hadrons with $c$ and $b$ quarks.

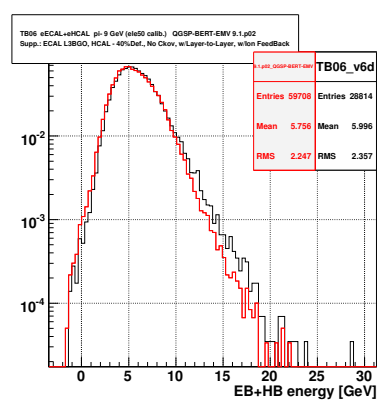

$9 \mathrm{GeV}$ pions

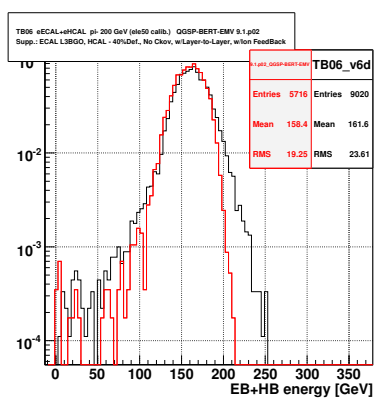

$200 \mathrm{GeV}$ pions
Fig. 10. Outstanding issues: Width of reconstructed energy.

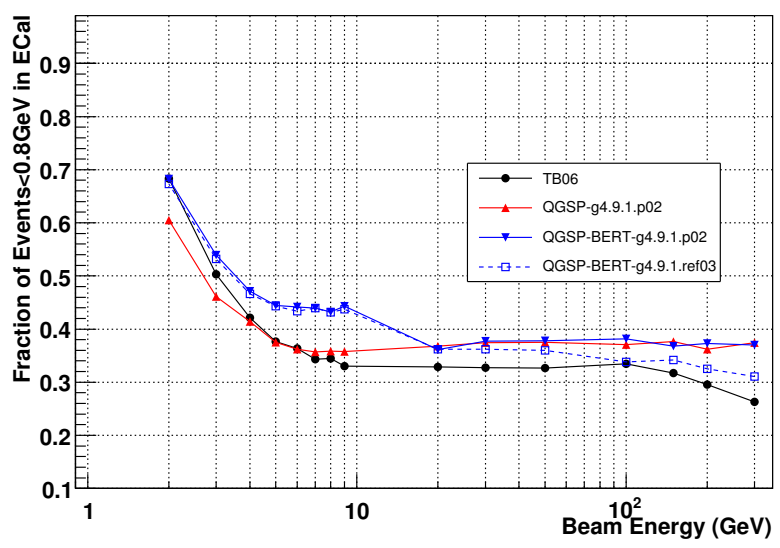

Fig. 11. Outstanding issues: Fraction of MIP-like events in ECAL

\section{RESULTS}

After these improvements in the simulation were introduced, the agreement between test-beam data and simulation was dramatically improved. Fig.4 shows the linearity of response for pions and protons over the whole momentum range for both the combined ECAL+HCAL system (left) and for HCAL alone (right). Fig.5 shows the resolution of the combined system (left) and HCAL alone (right). Longitudinal and transverse shower profiles (Fig.9 also show better agreement to data.

\section{OUTSTANDING ISSUES}

Even after these dramatic improvements in linearity of response and shower profiles, few issues are still present in the simulation which require further understanding and improvement. As we saw in Fig.5 the resolution of the simulated 
detector is much better at high momenta than the measured one. The reason for this is seen in Fig.10 which compares the distributions of the reconstructed energy in test-beam and simulation for two different beam momenta: $9 \mathrm{GeV} / \mathrm{c}$ (left) and $200 \mathrm{GeV} / \mathrm{c}$ (right). The simulated distribution agrees very well at low energies, but seems to be much narrower at high energies, resulting in the observed better resolution.

Another outstanding issue is the difference in the number of pions that cross the ECAL crystals without interacting hadronically, like Minimum Ionizing Particles (MIP), as seen in Fig.11. Bertini cascade shows a discontinuity in this quantity around $10 \mathrm{GeV} / \mathrm{c}$, which is the cut-off energy for that model. On the positive side, we see that the newest versions of G4 (9.1.ref03) include proper treatment of pion bremsstrahlung something that was missing in earlier versions.

\section{CONCLUSION}

CMS collaboration is putting a big effort into validating the Monte-Carlo simulation of its calorimetric system with dedicated test-beam data. Many of the observed discrepancies were removed by the use of Birks' law saturation in the scintillators, and by the introduction of new physics models for hadron interactions with matter.
There are still a few outstanding issues, which we hope to have resolved in collaboration with the Geant 4 team before the appearance of first LHC collision data.

\section{REFERENCES}

[1] The CMS Collaboration, Technical Proposal, CERN/LHCC 94-38, 1994

[2] R. Adolphi et al. [CMS Collaboration], JINST 3 (2008) S08004

[3] S. Agostinelli et al. [GEANT4 Collaboration], "GEANT4: A simulation toolkit," Nucl. Instrum. Meth. A 506 (2003) 250.

[4] J. Allison et al., "Geant4 developments and applications," IEEE Trans. Nucl. Sci. 53 (2006) 270.

[5] [CMS Collaboration], "CMS: The computing project. Technical design report," CERN-LHCC-2005-023

[6] G. Alverson, G. Eulisse, S. Muzaffar, I. Osborne, L. Taylor and L. A. Tuura, Nucl. Instrum. Meth. A 534, 143 (2004).

[7] http://geant4.cern.ch/support/index.shtml 